\title{
cAMP-PKA Signaling Regulates Multiple Steps of Fungal Infection Cooperatively with Cmk1 MAP Kinase in Colletotrichum lagenarium
}

\author{
Junko Yamauchi, Naoyuki Takayanagi, Kenichi Komeda, Yoshitaka Takano, and Tetsuro Okuno \\ Laboratory of Plant Pathology, Graduate School of Agriculture, Kyoto University, Kyoto, 606-8502, Japan
}

Submitted 24 May 2004. Accepted 26 July 2004.

\begin{abstract}
In Colletotrichum lagenarium, RPK1 encoding the regulatory subunit of PKA is required for pathogenicity. From the $r p k 1$ mutant that forms small colonies, we isolated three growth-suppressor mutants. All rpk1-suppressor mutants are nonpathogenic and contain amino acid changes in the PKA catalytic subunit Cpk1. To assess the roles of cyclic AMP (cAMP) signaling in detail, we generated knockout mutants of $C P K 1$ and the adenylate cyclase gene $C A C 1$. The cpk1 and cac1 mutants are nonpathogenic on cucumber. Interestingly, both of the mutants germinated poorly, suggesting involvement of cAMP signaling in germination. Germination defect in the $c p k 1$ and $c a c 1$ mutants is partially rescued by incubation of the conidia at lower concentrations. Germinating conidia of the cpk1 and cac1 mutants can form appressoria, but the appressoria formed by them are nonfunctional, like those of the rpk1 mutant. $\mathrm{Cy}$ tological analysis indicates that the appressoria of the $c p k 1$ mutant contain larger numbers of lipid bodies compared with the wild type, whereas lipid levels in the rpk1 mutants are lower, suggesting cAMP-mediated regulation of lipid metabolism for appressorium functionality. Furthermore, the cpk1 and cac1 mutants have a defect in infectious growth in plant. In $C$. lagenarium, Cmk1 mitogen-activated protein kinase (MAPK) regulates germination, appressorium formation, and infectious growth. These results suggest that cAMP signaling controls multiple steps of fungal infection in cooperative regulation with Cmk1 MAPK in $C$. lagenarium.
\end{abstract}

Colletotrichum spp. cause anthracnose diseases on numerous crops and ornamental plants (Agrios 1988). During the infection of plants, asexual spores (conidia) of Colletotrichum species germinate on the host plants and germ tubes differentiate into specialized infection structures called appressoria. Mitogen-activated protein kinase (MAPK) cascades play pivotal roles in fungal infection processes, including appressorium formation, in fungal pathogens (Xu 2000). In Colletotrichum lagenarium, which causes cucumber anthracnose, two MAPKs, Maf1 and Cmk1, regulate fungal pathogenesis. Deletion mutants of MAF1, which encodes a MAPK related to Saccharomyces cerevisiae Mpk1 (Slt2), show reduced pathogenicity on cucumber. Conidia of the mafl mutant germinate efficiently but fail to form appressoria, indicating that $M A F 1$ regulates appressorium differentiation during fungal infection (Kojima et al. 2002). $C M K 1$, which encodes a MAPK related to $S$. cerevisiae Fus3/Kss1, also

Corresponding author: Y. Takano; Telephone: 81-75-753-6132; Fax: 81-75753-6131; E-mail: ytakano@kais.kyoto-u.ac.jp is required for fungal pathogenicity on cucumber. Further phenotypic analysis of the $c m k 1$ mutants indicated that $C M K 1$ is involved in germination, appressorium formation, and infectious growth in planta (Takano et al. 2000). The cmkl mutant failed to germinate but the addition of yeast extract restored germination of the mutant, which strongly suggests the presence of an unidentified signaling pathway that regulates germination in addition to the Cmk1-mediated pathway.

The cyclic AMP (cAMP) signaling pathway also has been shown to play critical roles in the pathogenesis in several fungal pathogens (Kronstad 1997; Lee et al. 2003). In a maize pathogen, Ustilago maydis, cAMP signaling is involved in pheromone responses and fungal pathogenesis, including gall formation (Gold et al. 1994, 1997). In the rice blast fungus, Magnaporthe grisea, germinating conidia form appressoria efficiently on hydrophobic surfaces but not on hydrophilic surfaces (Lee and Dean 1994). cAMP induces appressorium formation on noninductive hydrophilic surfaces, suggesting the involvement of cAMP signaling in appressorium formation (Lee and Dean 1993). Consistent with this, disruption of the adenylate cyclase gene MAC1 in M. grisea caused defects in appressorium formation (Adachi and Hamer 1998; Choi and Dean 1997). On the other hand, mutants of $C P K A$, which encodes a PKA catalytic subunit, retained an ability to develop appressoria, although appressoria formed by the cpkA mutant are nonfunctional (Mitchell and Dean 1995; Xu et al. 1997). The PKA catalytic subunit mutants of $C$. trifolii also form appressoria but fail to penetrate (Yang and Dickman 1999). At present, the question remains whether cAMP signaling is a common regulatory system for appressorium formation in other fungal pathogens.

The PKA regulatory subunit gene RPKI of C. lagenarium was isolated and characterized. The rpkl deletion mutant, which exhibits high constitutive PKA activity, shows significant reduction in vegetative growth, mycelial melanization, and conidiation. The rpkl mutant lacks fungal pathogenicity, suggesting that hyperactivated PKA has negative effects on fungal pathogenicity (Takano et al. 2001a). We isolated a growth-suppressor mutant from the rpkl mutant. The suppressor mutant has lost most PKA activity. The rpkl-suppressor mutant is nonpathogenic on cucumber, like the parental rpkl mutant. These observations strongly suggest that a certain level of PKA regulated by Rpk1 is required for the pathogenicity of anthracnose fungi (Takano et al. 2001a). However, the impact of complete inactivation of cAMP signaling on the fungal pathogenesis of C. lagenarium remains to be elucidated. To better understand the role of cAMP signaling in fungal pathogenicity, we assessed roles of the PKA catalytic subunit gene $(C P K 1)$ and the adenylate cyclase gene $(C A C 1)$ in $C$. lagenarium in this article. Disruption of each gene results in loss of pathogenicity on cu- 
A

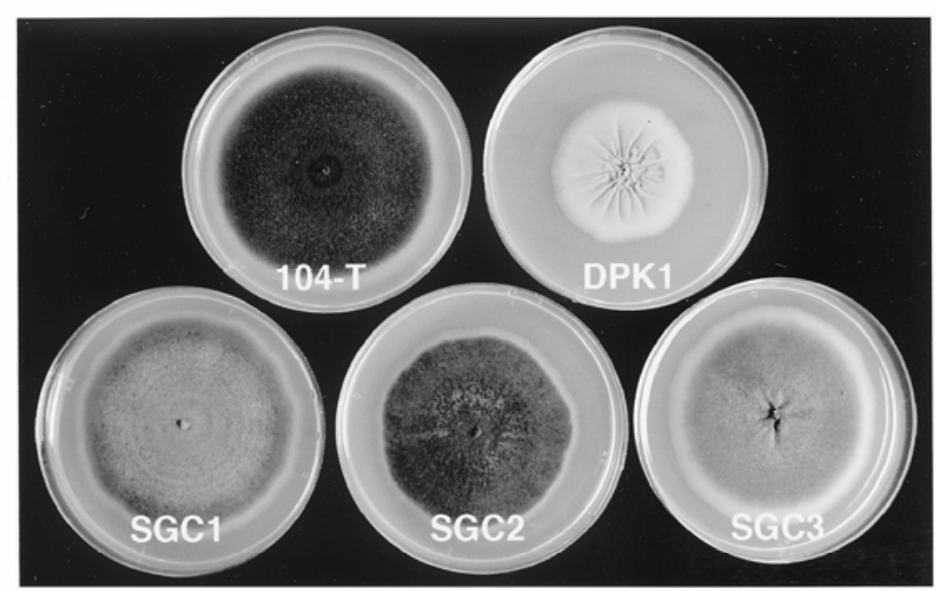

B

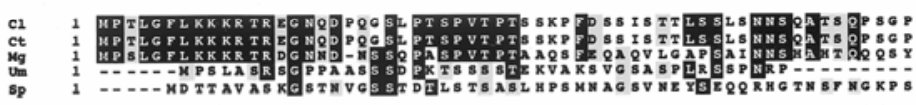

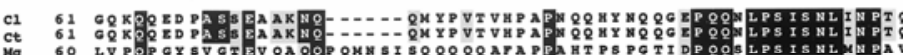

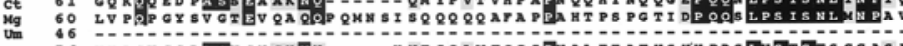

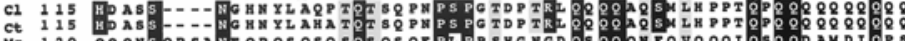

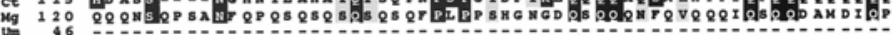

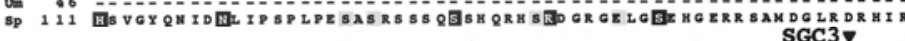

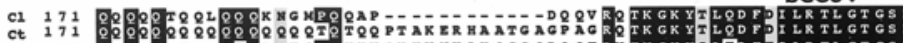

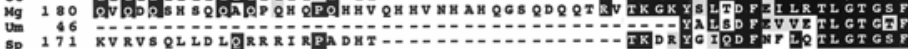

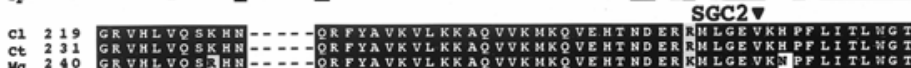

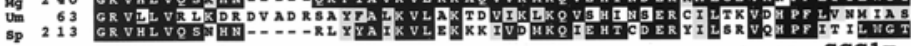

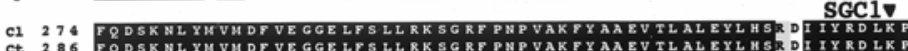

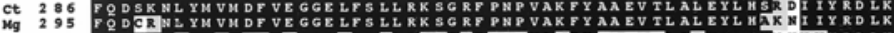

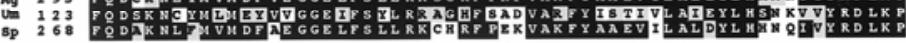

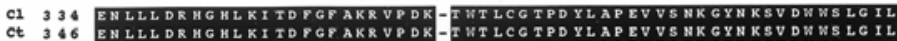

$M g$
$M$
Me

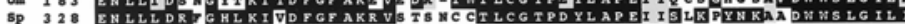

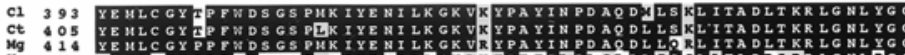

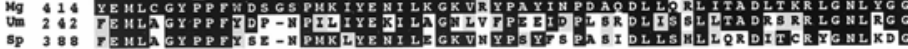

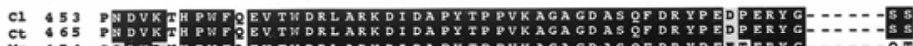

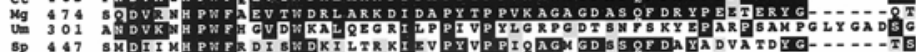

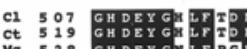

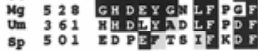

C

\begin{tabular}{|c|c|c|c|}
\hline Strain & Codon & Amino & acid sequence \\
\hline $\begin{array}{l}104-T \\
\text { SGC1 }\end{array}$ & $\begin{array}{l}A \underline{A} \bar{G} \\
A \underline{G} G\end{array}$ & $\begin{array}{l}325: \\
325:\end{array}$ & 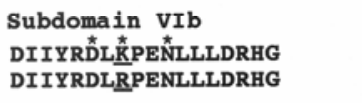 \\
\hline $\begin{array}{l}104-T \\
\text { SGC2 }\end{array}$ & $\begin{array}{l}\text { GTC } \\
\text { GAC }\end{array}$ & $\begin{array}{l}262: \\
262:\end{array}$ & $\begin{array}{l}\text { Subdomain III } \\
\text { QVEHTNDẼRRMLGEV } \\
\text { QVEHTNDERRMLGED }\end{array}$ \\
\hline $\begin{array}{l}104-T \\
\text { SGC3 }\end{array}$ & $\begin{array}{l}\mathrm{GGA} \\
\mathrm{GAA}\end{array}$ & $\begin{array}{l}\text { 207: } \\
\text { 207: }\end{array}$ & 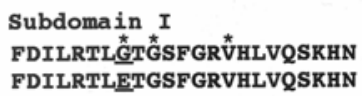 \\
\hline
\end{tabular}

Fig. 1. SGC mutants have mutations in CPK1. A, Colonies of wild-type and mutant strains. Wild-type strain 104-T, the rpk1 4 strain DPK1, and the $r p k 1$ suppressor mutants SGC1, SGC2, and SGC3 were incubated on potato dextrose agar at $24^{\circ} \mathrm{C}$ for 2 weeks. B, Amino acid sequence alignment of Cpk1 of Colletotrichum lagenarium $(\mathrm{Cl})$ with the primary sequences of the catalytic subunit of PKA from other organisms. Deduced amino acid sequence of Cpk1 was aligned with those of Ct-Pkac of C. trifolii (Ct), Cpka of Magnaporthe grisea (Mg), Adr1 of Ustilago. maydis (Um), and Pka1 of Schizosaccharomyces pombe (Sp) using the Clustal W program (Thompson et al. 1994). Identical amino acids are indicated as white letters on black ground. Similar residues are shown on a gray background. Gaps introduced for the alignment are indicated by hyphens. GenBank accession numbers for Ct-Pkac, Cpka, Adr1, and Pka1 are AF046921, U12335, AF025290, and D23667, respectively. Mutation points in Cpk1 of the SGC mutants are indicated by arrowheads. C, Amino acid changes found in subdomains of Cpk1 of the SGC mutants. Altered amino acids in the SGC mutants are underlined. Amino acids conserved in serine/threonine protein kinases are indicated by asterisks. 
cumber. Surprisingly, the cpkl and cacl mutants germinated poorly, suggesting that cAMP or PKA regulates germination. However, germination of the cpkl and cacl mutants was restored partially when conidial concentrations were lowered. Further analysis of the cpkl and cacl mutants suggested that cAMP-PKA signaling regulates appressorial functionality and infectious growth in plant, whereas cAMP-PKA signaling is not essential for morphological development of appressoria, unlike $M$. grisea. Our report highlights the novel contribution of cAMP signaling in the regulation of fungal infection processes, in part, in cooperation with the MAPK cascade.

\section{RESULTS}

\section{$R P K 1$ requirements for fungal development are suppressed by mutations in the PKA catalytic subunit.}

We have shown previously that the disruption of RPK1 encoding the PKA regulatory subunit caused pleiotropic effects in $C$. lagenarium, including a reduction in mycelial growth, melanization, conidiation, and fungal pathogenicity. From the rpkl $\Delta$ strain, a suppressor mutant SGC1 was isolated that exhibited increased growth, melanization, and conidiation compared with those functions of the parental rpkl $\Delta$ strain (Takano et al. 2001a) (Fig. 1A; Table 1). A PKA assay showed that SGC1 lacks most functional PKA activity, suggesting the possibility that SGC1 carries a mutation in the PKA catalytic subunit (Takano et al. 2001a). To assess this possibility, we isolated the PKA catalytic subunit gene from $C$. lagenarium and compared its sequence in the wild-type strain and SGC1. The PKA catalytic subunit gene of $C$. lagenarium, designated $C P K 1$, was isolated by polymerase chain reaction (PCR) with degenerate primers designed from amino acid sequences conserved in other fungal PKA catalytic subunits. The genome and cDNA sequences of $C P K 1$ indicated that $C P K 1$ consists of a 1,725-bp open reading frame (ORF) encoding a 518-aminoacid (aa) protein with three introns (Fig. 1B) (GenBank accession number AB127956). The deduced amino acid sequence of $C P K 1$ has high homology with the primary sequences of the PKA catalytic subunits of C. trifolii (96\% identity), M. grisea (69\%), U. maydis (48\%), and Schizosaccharomyces pombe (44\%) (Fig. 1B). Transcription of CPK1 in the wild type and SGC1 was investigated by reverse transcription (RT)-PCR. As a result, transcripts from $C P K 1$ were detected in $\mathrm{SGC1}$ as well as in the wild type (data not shown). To investigate whether SGC1 has a mutation in the $C P K 1$-encoded PKA catalytic subunit, the full length of $C P K 1$ was amplified from the genome of SGC1. Sequence analysis of the amplified fragment revealed that SGC1 contains a single nucleotide mutation in the $C P K 1$ coding sequence, and that the mutation causes an amino acid change. PCR amplification and sequence analysis were independently repeated twice, and identical results were obtained. In SGC1, an A-to-G mutation occurs at nucleotide position 1,052 of $C P K 1$ that changes Lys to Arg at amino acid residue 332 (Fig. 1B and C). The mutation of SGC1 in Cpk1 (cpk11 ) is located in the subdomain VIb of protein kinases, and the altered amino acid is highly conserved in the serine/threonine kinases (Hanks and Quinn 1991). To assess whether the mutation in $C P K 1$ is responsible for the SGC1 phenotype, we reintroduced the wild-type $C P K 1$ gene into SGC1. The plasmid pCBCPK1 carrying $C P K 1$ and a bialaphos-resistance (bar) gene was introduced into SGC1. Of 38 bialaphos-resistant pCBCPK1 transformants, 20 exhibited less melanized and small colonies which were similar to those formed by the rpklA strain, whereas all transformants of pCB1531, which carries the bar gene without $C P K 1$, showed the colony phenotype of SGC1. These results demonstrated that the point mutation in $C P K 1$ generated SGC1 from the rpkl $\triangle$ strain.
We also isolated two additional suppressor mutants from the rpk1 1 strain, designated SGC2 and SGC3 (Fig. 1A). Both SGC2 and SGC3 showed increased growth, melanization, and conidiation compared with the rpkl $\Delta$ strain, although the number of conidia in SGC2 was less than in SGC1 or SGC3 (data not shown). To assess the possibility that SGC2 and SGC3 have a mutation in $C P K 1$, we amplified genomic fragments containing $C P K 1$ from SGC2 and SGC3 and sequenced them. Notably, both SGC2 and SGC3 have distinct single-nucleotide mutations in $C P K 1$ that alter amino acid residues. In SGC2, a T-to-A mutation occurs at nucleotide position 785 that changes Val to Asp. The mutation point of SGC2 is located in the subdomain III of protein kinases (Fig. 1B and C). In SGC3, a Gto-A mutation occurs at nucleotide position 641 that changes Gly to Glu. The mutation of SGC3 is located in subdomain I of protein kinases, and the changed amino acid residue is highly conserved in the catalytic domains of the serine/threonine kinases. An inoculation assay with SGC2 (rpkl $1, c p k 1-2)$ and SGC3 (rpkl,$c p k 1-3)$ showed that both mutants are nonpathogenic on cucumber, like SGC1 (data not shown). We investigated the infection-related morphogenesis of the SGC mutants. On a glass surface, the SGC mutants commonly showed reduction in the germination frequency of conidia. In the wild-type strain 104-T, $90.0 \pm 3.3 \%$ of conidia germinated within $12 \mathrm{~h}$ of incubation. In contrast, germination of SGC1, SGC2, and SGC3 was $25.0 \pm 2.7,57.0 \pm 17$, and $40 \pm 3.3 \%$, respectively, implying the involvement of $C P K 1$ in germination. Germinating conidia of all SGC mutants formed melanized appressoria (data not shown).

Inactivation of genes involved in cAMP signaling pathways.

Analysis of the SGC mutants strongly suggested the involvement of $C P K 1$ in the fungal pathogenicity of $C$. lagenarium. Because i) the phenotypes of the three SGC mutants are not completely identical and ii) the SGC mutants lack the $R P K 1$ gene as well as containing the $C P K 1$ mutations, we generated a null mutant of $C P K 1$ to precisely assess the function of $C P K 1$ in $C$. lagenarium. A gene disruption vector, pGDCPK1, which contains a hygromycin B phosphotransferase $(h p h)$ gene cassette and both $5^{\prime}$ and $3^{\prime}$ flanking regions of $C P K 1$, was constructed, and introduced into the wild-type strain 104-T (Fig. 2A). Of 32 hygromycin-resistant transformants, 30 transformants displayed reduced colony size relative to that of 104-T. Using DNA gel blot analysis, we investigated whether $C P K 1$ is disrupted in the transformants that showed reduced growth. Genomic DNAs were isolated from 104-T and three transformants, DCP3, DCP11, and DCP17, that showed reduced growth. Isolated genomic DNAs were digested with ApaI and probed with the 2.0-kb KpnI-ApaI fragment from the $C P K 1$ locus (Fig. 2B). 104-T contained the 7.0-kb ApaI fragment. On the other hand, DCP3, DCP11, and DCP17 did not generate the 7.0-kb ApaI fragment, but contained a common 5.0-kb ApaI

Table 1. Strains used in this study

\begin{tabular}{lll}
\hline Strain & Genotype & \multicolumn{1}{c}{ Reference } \\
\hline 104-T & Wild type & Ishida and Akai 1969 \\
DPK1 & $r p k 1 \Delta$ & Takano et al. 2001 \\
DCM1 & $c m k 1 \Delta$ & Takano et al. 2000 \\
ALB1 & $p k s 1:: h p h$ & This study \\
SGC1 & $r p k 1 \Delta, c p k 1-1$ & Takano et al. 2001 \\
SGC2 & $r p k 1 \Delta, c p k 1-2$ & This study \\
SGC3 & $r p k 1 \Delta, c p k 1-3$ & This study \\
DCP3 & $c p k 1 \Delta$ & This study \\
DCP11 & $c p k 1 \Delta$ & This study \\
DRC1 & $r p k 1 \Delta, c p k 1 \Delta$ & This study \\
DCA1 & $c a c 1:: h p h$ & This study \\
DCA2 & $c a c 1:: h p h$ & This study \\
\hline
\end{tabular}


fragment, which is consistent with the expected length after a gene replacement event. We conclude that $C P K 1$ is disrupted in DCP3, DCP11, and DCP17. The cpk1 $\Delta$ strains DCP3 and DCP11 were subjected to further analysis.

In addition to analysis of $C P K 1$, we investigated the function of adenylate cyclase to better understand the role of cAMP signaling in $C$. lagenarium. Using PCR with degenerate primers, we isolated an adenylate cyclase gene, designated $C A C 1$ from C. lagenarium. Sequencing of the genomic fragment of $C A C 1$ identified a 7,003-bp ORF with three predicted introns, which putatively encodes a 2,143-aa polypeptide (GenBank accession no. AB127957). The deduced amino acid sequence of $C A C 1$
A

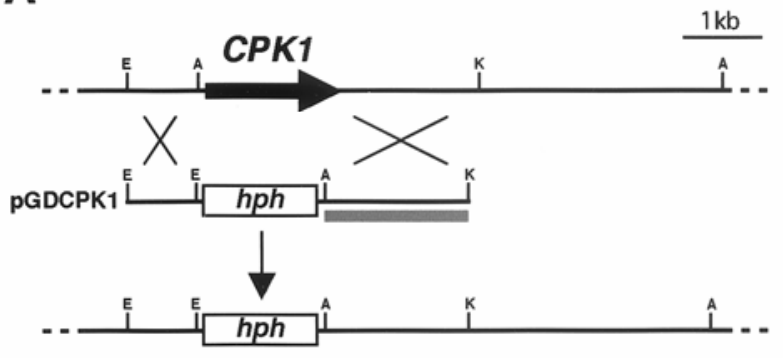

C
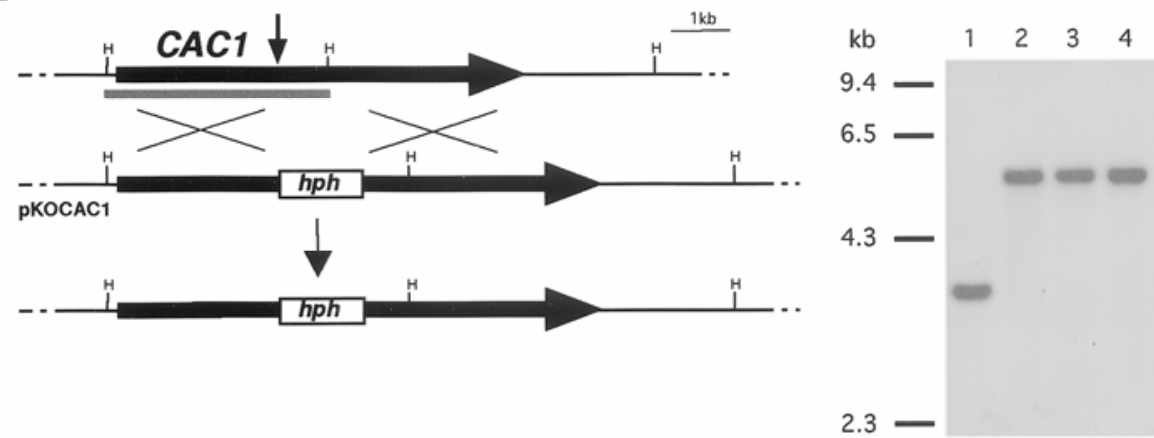

E

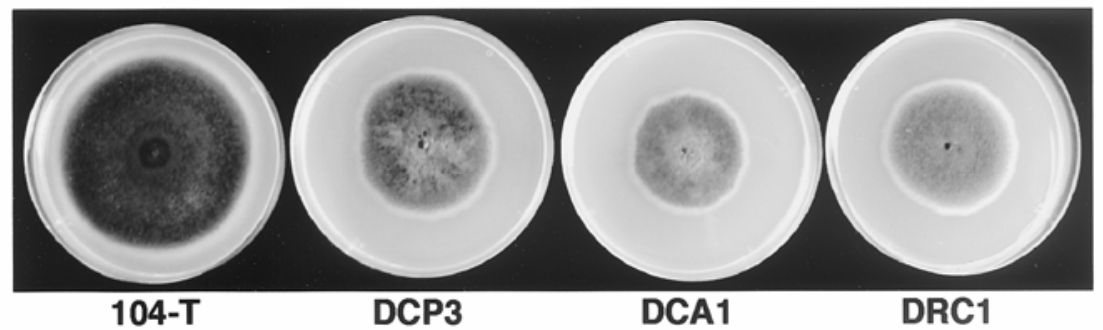

$\mathbf{F}$

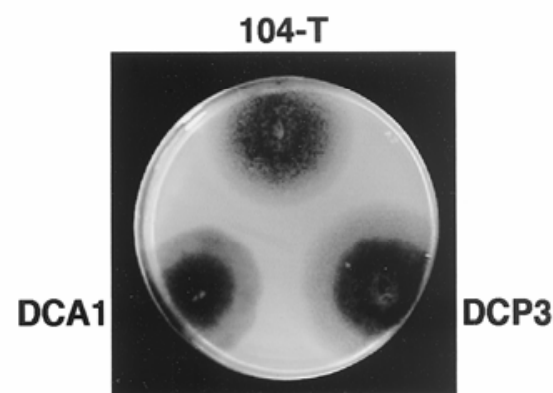

Fig. 2. Gene disruption of $C P K 1$ and $C A C 1$. A, $C P K 1$ locus and the gene replacement vector pGDCPK1. pGDCPK1 contains a hygromycin phosphotransferase gene $(h p h)$ cassette flanked by border sequences from CPK1. A, ApaI; E, EcoRI; K, KpnI. B, DNA gel blot analysis of the CPK1 gene replacement mutants. Genomic DNAs were isolated from the wild-type strain 104-T (lane 1), and gene replacement transformants DCP3, DCP11, and DCP17 (lanes 2 to 4, respectively). The genomic DNAs were digested with ApaI. The blot was hybridized with a 2.0-kb KpnI-ApaI fragment, indicated by the gray bar in A. C, CACl locus and gene disruption vector pKOCAC1. The GPS-HYG insertion site is indicated by arrow. H, HindIII. D, DNA gel blot analysis of the CAC1 gene replacement mutants. Genomic DNAs were isolated from the wild-type strain 104-T (lane 1), and CAC1 gene disruption transformants DCA1, DCA2, and DCA8 (lanes 2 to 4, respectively). The genomic DNAs were digested with HindIII. The blot was hybridized with a 3.6-kb HindIII fragment, indicated by the gray bar in C. E, Mycelial growth of the cyclic AMP (cAMP) signaling mutants on potato dextrose agar (PDA). The wildtype strain 104-T, the $c p k 1 \Delta$ strain DCP3, the $c a c 1:: h p h$ strain DCA1, and the $r p k 1 \Delta$ cpk1 1 strain DRC1 were incubated on PDA at $24^{\circ} \mathrm{C}$ for 2 weeks. F, Growth of the cAMP signaling mutants on minimal medium. Each strain was incubated on minimal medium at $24^{\circ} \mathrm{C}$ for 2 weeks. 
shares significant homology with those of other fungal adenylate cyclase genes: $M$. grisea MAC1 (66\% identity), Neurospora crassa CR-1 (56\%), and Saccharomyces cerevisiae CYRI (32\%). Targeted gene disruption of $C A C l$ was performed using an in vitro transposon tagging procedure, called TAG-KO, as previously reported (Hamer et al. 2001). The modified Tn7 transposable element containing the hygromycin-B-resistance gene cassette (the GPS-Hyg transposon) was mobilized to the CACl cosmid clone in vitro. A gene disruption construct, pKOCAC1, containing CACl interrupted by the GPS-Hyg transposon, was identified. In pKOCAC1, the transposon was inserted at nucleotide 2,877 (amino acid residue 788) of $C A C 1$. pKOCAC1 was introduced into 104-T and transformants were selected with hygromycin. All 20 hygromycin-resistant transformants exhibited reduced growth, and 3 of them were investigated for gene replacement events in $C A C 1$ by DNA gel blot analysis (Fig. 2C and D). The genomic DNA of each transformant was digested with HindIII and probed with a 3.6-kb PCR fragment containing $C A C 1$. Transformants DCA1, DCA2, and DCA8 did not contain the 3.6-kb HindIII fragment detected in 104-T but contained a common 5.1-kb HindIII fragment, which was consistent with the expected length for the gene disruption event. We conclude that $C A C 1$ is disrupted in DCA1, DCA2, and DCA8. The cacl::hph strains DCA1 and DCA2 were subjected to further analysis.

The cpkls strains (DCP3 and DCP11) and the cacl::hph strains (DCA1 and DCA2) showed reduction in vegetative growth on potato dextrose agar (PDA) medium (Fig. 2E; Table 2 ). The linear growth rates of the $c p k l \Delta$ and $c a c 1:: h p h$ strains were reduced to 70 and $60 \%$, respectively, of 104-T. The number of conidia produced by the cpkl $\Delta$ and cacl strains was reduced to approximately 35 and $10 \%$, respectively, of that of 104-T. These results suggest that cAMP signaling is required by $C$. lagenarium for vegetative growth and conidiation on nutrient rich medium. When the $c a c l$ strains were grown on PDA

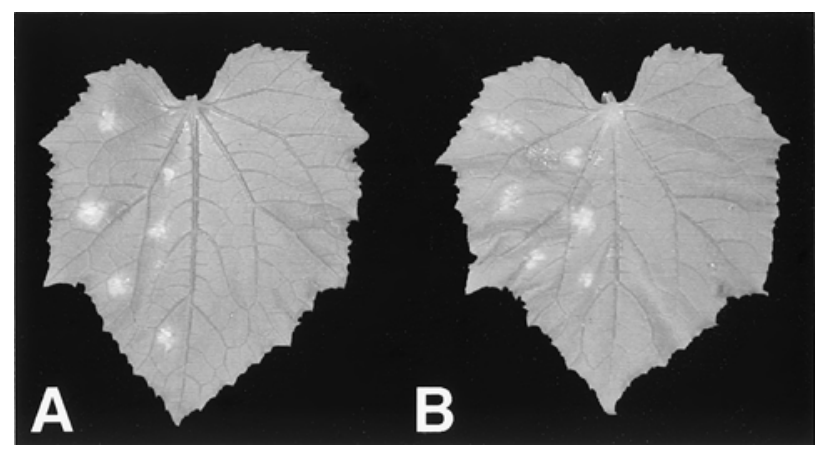

Fig. 3. $C P K 1$ and $C A C 1$ are essential for pathogenicity. Conidial suspensions of tested strains were drop inoculated onto detached cucumber leaves, and inoculated leaves were incubated at $24^{\circ} \mathrm{C}$ for 10 days. On the left half of the leaves, drops of conidial suspension of the wild-type strain 104-T were inoculated as a positive control. On the right half of the leaves, $\mathbf{A}$, the cpk1 $\Delta$ strain DCP3 and $\mathbf{B}$, the cac1::hph strain DCA1 were inoculated. with $5 \mathrm{mM}$ cAMP, their growth and conidiation were restored, whereas those of the $c p k 1 \Delta$ strains were not (Table 2). This suggests that extracellular cAMP can restore mycelial growth and conidiation of the $c a c l$ strains. The $c p k l \Delta$ and $c a c l$ strains did not show reduction in growth rate on minimal media (MM) (i.e., growth rate of the cpkl $\Delta$ and cacl strains was 122 and 95\%, respectively, of 104-T) (Fig. 2F).

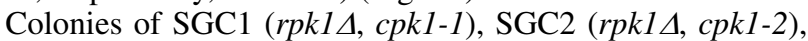
and SGC3 (rpkl $\Delta, c p k 1-3)$ are larger than those of the $c p k 1 \Delta$ strains on PDA. This result raises the possibility that differences between the cpkl $\Delta$ and SGC mutants are due to i) a deletion effect of RPKI in the SGC mutants or ii) different $C P K 1$

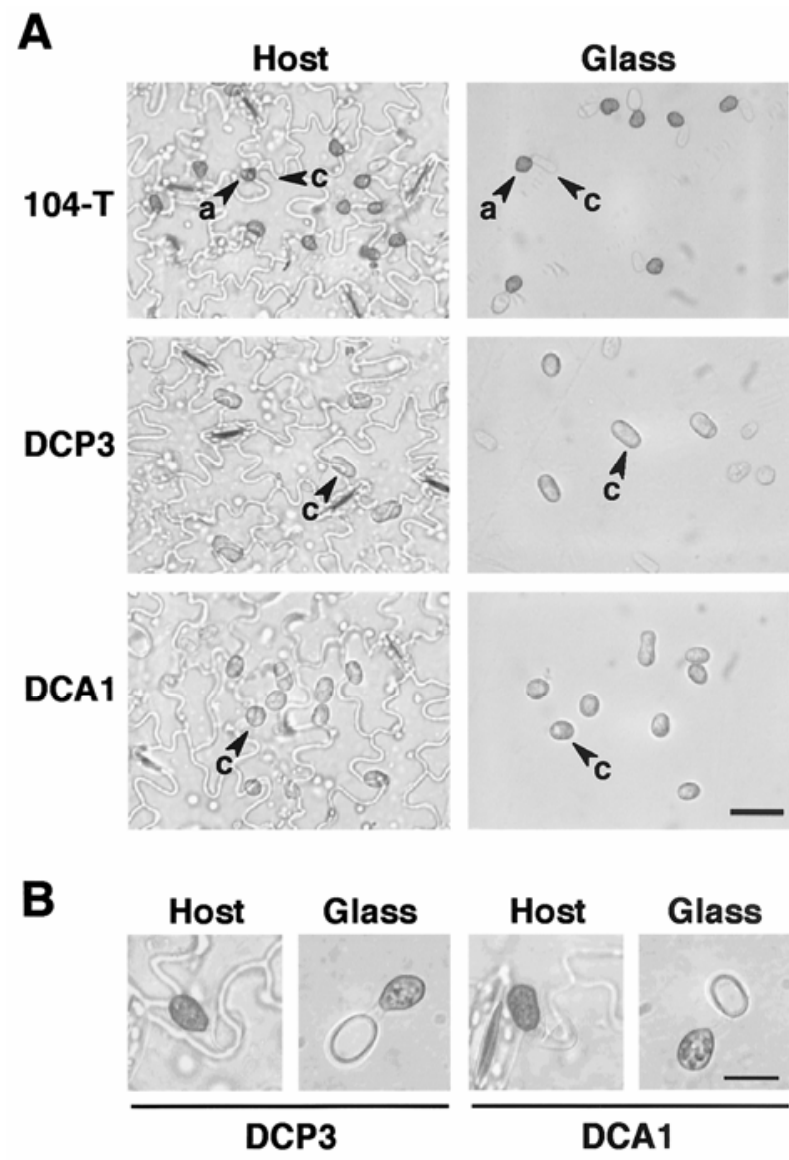

Fig. 4. Conidial germination of the cyclic AMP (cAMP)-signaling mutants. A, Failure of germination of the $c p k 1$ and cacl strains. Conidia of the wild-type strain 104-T, the cpk1 $\Delta$ strain DCP3, and the cacl strain DCA1 were incubated at $24^{\circ} \mathrm{C}$ for $24 \mathrm{~h}$ on host surfaces or glass surfaces at $10^{5}$ conidia/ml; a, appressorium; c, conidium. Bar $=20 \mu \mathrm{m}$. B, Appressoria formed by the cAMP-signaling mutants. At low conidial density, conidia of the $c p k 1$ and $c a c 1$ strains partially germinated and the germ tubes differentiated into appressoria. Conidia of each strain were incubated at $24^{\circ} \mathrm{C}$ for $24 \mathrm{~h}$ on host surface or glass surface at $10^{4}$ conidia $/ \mathrm{ml}$. Bar $=$ $10 \mu \mathrm{m}$.

Table 2. Growth and conidiation in the $c p k 1$ and $c a c 1$ mutants on potato dextrose agar

\begin{tabular}{|c|c|c|c|c|}
\hline \multirow[b]{2}{*}{ Strain $^{c}$} & \multicolumn{2}{|c|}{ Vegetative growth $(\mathrm{mm})^{\mathrm{a}}$} & \multicolumn{2}{|c|}{ Conidia $\left(\times 10^{6}\right)^{b}$} \\
\hline & No cAMP & 5 mM cAMP & No cAMP & 5 mM cAMP \\
\hline $104-\mathrm{T}$ & $38.3 \pm 0.5$ & $37.6 \pm 0.2$ & $45.4 \pm 2.2$ & $28.8 \pm 0.5$ \\
\hline DCP3 & $28.8 \pm 0.2$ & $27.8 \pm 0.2$ & $16.7 \pm 5.8$ & $18.7 \pm 1.7$ \\
\hline DCP11 & $26.5 \pm 1.7$ & $27.8 \pm 0.5$ & $15.8 \pm 5.0$ & $16.7 \pm 3.6$ \\
\hline DCA1 & $23.1 \pm 0.2$ & $34.8 \pm 1.0$ & $3.2 \pm 0.8$ & $35.0 \pm 8.7$ \\
\hline DCA2 & $23.0 \pm 0.5$ & $34.3 \pm 0.7$ & $5.8 \pm 1.8$ & $34.7 \pm 18.6$ \\
\hline
\end{tabular}

${ }^{a}$ Strains were grown on potato dextrose agar (PDA) or PDA containing $5 \mathrm{mM}$ cyclic AMP (cAMP) at $24^{\circ} \mathrm{C}$ for 7 days, and colony diameter was measured.

${ }^{\mathrm{b}}$ Conidia were collected from 7-day-old culture and counted. Each value represents mean \pm standard deviation from three independent experiments.

${ }^{c}$ Strains: 104-T is the wild-type, DCP3 and DCP11 are the cpk1 $\Delta$ strains, and DCA1 and DCA2 are the cacl strains. 
mutations in the cpkl $\Delta$ strain and SGC mutants. To verify this,

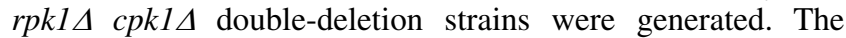
rpk1 1 mutant, DPK1, was co-transformed with the CPK1 knockout vector pGDCPK1 and pCB1531. Among the bialaphos-resistant DPK1 transformants, transformant DRC1 was confirmed to have a gene replacement for $C P K 1$ (data not shown). We found that the colony phenotype of DRC1 (rpklA cpkl $\Delta$ ) was very similar to that of the $c p k 1 \Delta$ strains (Fig. 2E). This indicates that deletion of $C P K 1$ in the $r p k 1 \Delta$ strain does not generate the SGC phenotype, and suggests that the different phenotypes of the cpkl $\Delta$ strain and SGC mutants mainly are due to differences in the $C P K 1$ mutations they contain.

\section{cAMP signaling is required for fungal pathogenicity.}

To assess the fungal pathogenicity of the $c p k l \Delta$ and $c a c l:: h p h$ strains, infection assays against the host plant (cucumber) were performed. Conidial suspensions of the wild-type 104-T, the cpkl $\Delta$ strain DCP3, and the cacl strain DCA1 were spotted onto detached host cucumber leaves and incubated (Fig. 3). The wild type formed lesions on inoculated leaves after 7 days. In contrast, DCP3 and DCA1 did not form any lesions. After 2 weeks, the yellowish lesions caused by 104-T spread and turned brown, whereas no lesions were observed on leaves inoculated with DCP3 or DCA1. Identical results were observed for inoculations with the two additional cpkl $\Delta$ and $c a c l$ strains tested. These results show that both $C P K 1$ and $C A C l$ are essential for the fungal pathogenicity of $C$. lagenarium.

\section{cAMP-PKA signaling regulates conidial germination.}

To investigate the deficient steps in the fungal pathogenicity of the $c p k 1 \Delta$ and $c a c l:: h p h$ strains, we examined the infection behavior of these mutants on host plants using microscopy. Conidial suspensions $\left(10^{5}\right.$ conidia/ml $)$ were spotted onto the lower surfaces of cucumber cotyledons and incubated. In the wild type, most conidia ( $>90 \%$ ) germinated and developed darkly melanized appressoria within $12 \mathrm{~h}$ (Fig. 4A). In contrast, the cpkl $\Delta$ strain DCP3 and the cacl::hph strain DCA1 did not germinate on host leaves after incubation for $12 \mathrm{~h}$. They germinated very poorly even after $24 \mathrm{~h}$ (Fig. 4A). The germination rates of both $c p k 1 \Delta$ and $c a c l:: h p h$ strains were $<5 \%(n=500)$ at $24 \mathrm{~h}$. This result suggests that $C A C l$ and $C P K 1$ play important roles in germination. The cpkl $\triangle$ and cacl strains also germinated poorly on glass even after $24 \mathrm{~h}$, whereas germination of the wild type was complete by $12 \mathrm{~h}$ (Fig. 4A; Table 3). Similar results were observed in an additional two $c p k 1 \Delta$ and $c a c l$ strains tested. Thus, failure of germination is an inherent defect in the cpkl $\Delta$ and cacl strains. When conidia of the cacl strain were incubated in $50 \mathrm{mM}$ cAMP solution for $24 \mathrm{~h}$, the germination frequency of the $c a c l$ strains increased significantly (Table 3 ), suggesting that the addition of extracellular cAMP at the start of conidial incubation can rescue the germination defect of the $\mathrm{cacl}$ strain.

In C. lagenarium, CMK1-encoded MAPK is involved in germination of conidia (Takano et al. 2000). The cmkl $\Delta$ strains also have a decreased capacity for conidial attachment on hard surfaces (Takano et al. 2000). We measured conidial attachment of the $c p k l$ and $c a c l$ mutants to glass surfaces. In this assay, $86 \%$ of the wild-type conidia remained attached to the glass surface. Of the cpkl $\Delta$ strain and $c a c l$ strain conidia, 52 and $68 \%$, respectively, remained attached to the glass surface, whereas approximately $30 \%$ of conidia of the $\mathrm{cmkl} \Delta$ strains remained attached. Thus, inactivation of cAMP signaling slightly reduces the capacity for conidium attachment.

\section{Restored germination \\ in cAMP signaling and MAPK mutants.}

It has been reported that germination of the $\mathrm{cmkl} \Delta$ strain was rescued by the addition of $0.1 \%$ yeast extract (Takano et al. 2000). We investigated whether yeast extract can rescue the germination defects of the cpkl $\Delta$ and cacl strains. After $24 \mathrm{~h}$ of incubation in $0.1 \%$ yeast extract solution, the germination frequencies of the $c p k 1 \Delta$ and $c a c l$ strains were 8.9 and $1.9 \%$, respectively. In contrast, $>90 \%$ of conidia of the $c m k 1 \Delta$ strain germinated in $0.1 \%$ yeast extract solution (Table 3 ). Thus, yeast extract is less stimulating of germination in the cpkl $\Delta$ and $c a c l$ strains compared with that of the $c m k l \Delta$ strain.

The effects of carbon sources on the germination of the cpkl and $c a c l$ strains also were investigated. Conidia of the tested strains were incubated in $0.5 \%$ glucose solution or $0.5 \%$ fatty acid (Tween 80) solution. Neither glucose nor fatty acids has any effect on germination of the wild type (Table 2; data not shown). The cpkl and cacl strains failed to germinate with or without glucose (data not shown). However, addition of $0.5 \%$ Tween 80 increased the germination frequency of the cpkl $\Delta$ and cacl strains to 23.3 and $3.1 \%$, respectively. Germination of the cmkl strain also was restored by Tween 80 (Table 3). These data indicate that Tween 80 can partially restore the germination defects of the $c p k l \Delta, c a c l$, and $c m k l \Delta$ strains.

Interestingly, the $c p k l \Delta$ and $c a c l$ strains showed increased germination frequencies on both host and glass surfaces at a lower conidial density $\left(10^{4} \mathrm{conidia} / \mathrm{ml}\right)$ compared with the normal conidial density $\left(10^{5}\right.$ conidia/ml $)$. On the host surface, approximately $40 \%$ conidia of the $c p k l \Delta$ strain and $20 \%$ conidia of the cacl strain germinated at $24 \mathrm{~h}$ (Fig. 4B). On glass, the germination frequencies of the cpkl $\Delta$ and cacl strains at $10^{4}$ conidia/ml reached 30.7 and $3.3 \%$, respectively, (Table 3; Fig. 4B). In the presence of Tween 80, restoration of germination at the lower conidial density was more obvious in the $c a c l$ strain. In comparison with the cAMP signaling mutants, the $c m k 1 \Delta$ strains did not show obviously increased germination frequencies at $10^{4}$ conidia/ml (Table 3 ).

\section{Relationship of cAMP signaling to lipid metabolism in appressoria.}

Germinating conidia of the cpkl $\Delta$ and cacl strains, incubated at low densities, formed melanized appressoria on both plants and glass. This observation suggests that cAMP signaling is not essential for appressorium differentiation in $C$. lagenarium (Fig. 4B). However, when conidia of the cpkl $\Delta$ and cacl strains were inoculated on cucumber leaves at low den-

Table 3. Conidial germination of the cyclic AMP (cAMP) and the mitogen-activated protein kinase mutants ${ }^{\mathrm{a}}$

\begin{tabular}{|c|c|c|c|c|c|c|c|}
\hline \multirow[b]{2}{*}{ Strain } & \multicolumn{2}{|c|}{ No additive } & \multicolumn{2}{|c|}{$0.1 \%$ Yeast extract } & \multicolumn{2}{|c|}{$0.5 \%$ Tween 80} & \multirow{2}{*}{$\frac{50 \mathrm{mM} \text { cAMP }}{10^{5}}$} \\
\hline & $10^{5}$ & $10^{4}$ & $10^{5}$ & $10^{4}$ & $10^{5}$ & $10^{4}$ & \\
\hline 104-T (WT) & $94.3 \pm 1.8$ & ND & $99.5 \pm 0.5$ & ND & $95.1 \pm 6.7$ & ND & $96.6 \pm 0.8$ \\
\hline DCP3 (cpkl) & $5.4 \pm 1.8$ & $30.7 \pm 10.9$ & $8.9 \pm 2.2$ & $48.1 \pm 0.5$ & $23.3 \pm 4.4$ & $82.9 \pm 5.5$ & $2.9 \pm 1.6$ \\
\hline DCA1 (cacl) & $0.1 \pm 0.2$ & $3.3 \pm 3.2$ & $1.9 \pm 1.8$ & $14.9 \pm 5.8$ & $3.1 \pm 1.0$ & $77.9 \pm 9.9$ & $69.1 \pm 9.3$ \\
\hline DCM1 (cmkl) & $0.5 \pm 0.5$ & $2.5 \pm 1.6$ & $92.7 \pm 1.8$ & ND & $40.7 \pm 12.9$ & $64.3 \pm 3.8$ & $8.4 \pm 1.8$ \\
\hline
\end{tabular}

${ }^{a}$ Conidia were incubated at $10^{5}$ or $10^{4}$ conidia/ml. Conidia were incubated in glass petri dishes at $24^{\circ} \mathrm{C}$ for $24 \mathrm{~h}$. In each experiment, at least 200 conidia were examined to calculate percentage of germination. The mean and standard deviation were calculated form three independent experiments; ND = not determined. 
sity, each strain failed to form lesions, whereas the wild-type did form lesions. Appressoria developed by the $c p k 1 \Delta$ and cacl strains at low density failed to form penetration hyphae into the plants at 4 days postinoculation, although the wild type penetrated efficiently (data not shown). Consistent with this, all SGC mutants carrying mutated $C P K 1$ also failed to form penetration hyphae, suggesting that they have defects in appressorial penetration (data not shown). These results strongly suggest that cAMP or Cpk1 signaling is involved in the appressorial penetration step. The rpkl $\Delta$ strain of $C$. lagenarium also lacks the ability to form penetration hyphae (Takano et al. 2001a). These findings suggest that proper regulation of Cpk1 and Rpk1 via cAMP is required for appressorium function.

A
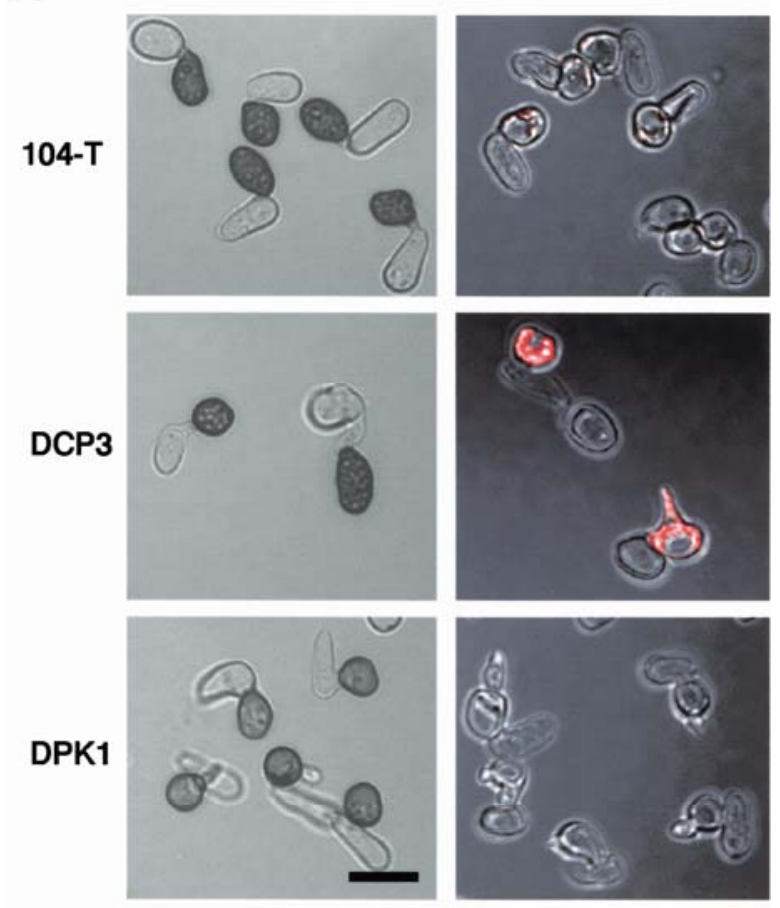

B

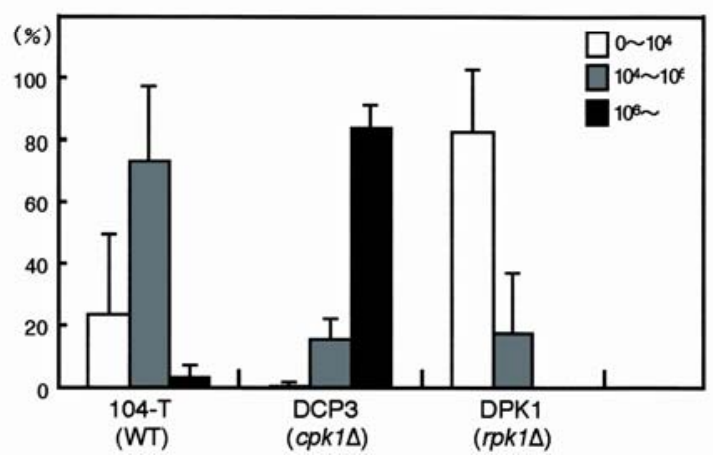

Fig. 5. Lipid bodies in appressoria. A, Conidia of the wild-type strain 104$\mathrm{T}$, the cpk1 $\Delta$ strain DCP3, and the rpk1 $\Delta$ strain DPK1 were incubated on glass slide at $24^{\circ} \mathrm{C}$ for $24 \mathrm{~h}$ (left panels). Conidia of tested strains were incubated on a glass slide with the melanin biosynthesis inhibitor carpropamid for $24 \mathrm{~h}$, and stained with Nile Red to visualize lipid bodies (right panels). Bar $=10 \mu \mathrm{m}$. B, Quantitative analysis of lipid bodies in appressoria. Conidia of 104-T, DCP3, and DPK1 were incubated for $24 \mathrm{~h}$ and stained with Nile Red. In each experiment, fluorescence of Nile Red in the appressoria of each strain $(n=50)$ was measured. Three categories of fluorescence were defined $\left(<10^{4}, 10^{4}\right.$ to $10^{6}$, and $>10^{6}$ intensity unit), and the proportion of each strain in each category was calculated. Means and standard deviations were calculated from three independent experiments.

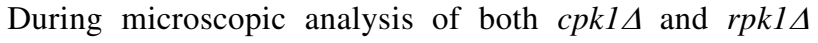
strains, we noticed that the number of lipid bodies inside the appressoria differed between the wild type and these mutants. Pre-incubated conidia of $C$. lagenarium contain large numbers of lipid bodies that can be stained by Nile Red. Conidia partially degrade lipid bodies in the appressorium-formation process, but newly formed appressoria still contain signifycant numbers of lipid bodies derived from their parental conidia. The lipid bodies in appressoria are degraded gradually, and this is followed by appressorial penetration. Appressoria of the wild-type 104-T strain retained lipid bodies at $24 \mathrm{~h}$, although degradation of lipid bodies had occurred (Fig. 5A). In contrast, no apparent reduction in lipid bodies was observed in the appressoria of the cpkl strain DCP3 at $24 \mathrm{~h}$, and, conversely, few lipid bodies remained in appressoria of the rpkl $\Delta$ strain DPK1 (Fig. 5A). To quantify the amount of lipid bodies in the appressoria of each strain, we stained them with Nile Red and measured relative Nile Red fluorescence inside the appressoria. Because Nile Red is not incorporated into melanized appressoria efficiently, the conidia were incubated with a melanin-biosynthesis inhibitor, carpropamid (Hattori et al. 1994), and the nonmelanized appressoria then were stained with Nile Red (Fig. 5A). Quantitative analysis of relative fluorescence value showed that $>70 \%$ of appressoria in the wild type showed fluorescence for Nile Red of $10^{4}$ to $10^{6}$ intensity unit, whereas $>80 \%$ of appressoria in the cpkl $\Delta$ strain showed fluorescence of $>10^{6}$ (Fig. 5B). In contrast, $>80 \%$ of appressoria of the $r p k 1 \Delta$ strain had fluorescence of $<10^{4}$ intensity unit (Fig. 5B). Therefore, deletion of CPK1 retarded the degradation of lipid bodies in the appressoria, whereas deletion of RPKI enhanced it. Together with these results, the finding that the appressoria of both the $c p k l$ and rpkl strains are not functional suggests that cAMP-PKA signaling regulates the metabolism of lipids in appressoria, and that this metabolic regulation might be involved in apressorium function in C. lagenarium.

cAMP signaling is required for infectious growth.

To assess the involvement of cAMP signaling in the infectious-growth stage, we used an inoculation assay for the $c p k 1$
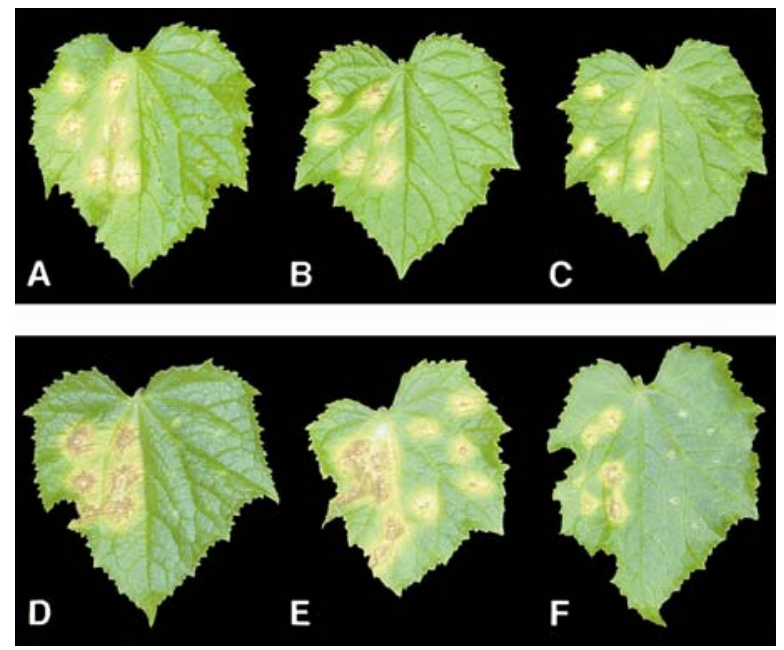

Fig. 6. Inoculation assays of wounded cucumber leaves. Mycelial blocks, prepared from fungal cultures on nutrient agar, were inoculated onto wound sites on detached cucumber leaves. On the left half of the cucumber leaves, mycelial blocks of the albino mutant ALB1 (pks $1:: h p h)$ were placed on the wound sites as a positive control. Mycelial blocks of tested strains were placed on the wound sites on the right half of the leaves. Inoculated leaves were incubated at $24^{\circ} \mathrm{C}$ for 10 days. A, DCP3 (cpk14); B, DCA1 (cacl::hph); C, DCM1 (cmk14); D, SGC1 (rpklA, $c p k 1-1) ; \mathbf{E}, \mathrm{SGC} 2(r p k 1 \Delta, c p k 1-2) ; \mathbf{F}, \mathrm{SGC} 3$ (rpk1 $1, c p k 1-3)$. 
and $c a c l$ strains through wound sites on cucumber leaves. Because the cpkl and cacl strains failed to germinate, mycelia were used for this assay instead of conidia. The pksl strain, which has a defect in melanin biosynthesis, lacks appressorial penetration ability but can grow invasively inside plants (Takano et al. 1995, 2000). When mycelial blocks of the $p k s 1:: h p h$ strain ALB1, generated by TAG-KO, were obtained from culture on PDA and inoculated onto wound sites on cucumber leaves, they formed lesions efficiently (Fig. 6). In C. lagenarium, CMK1 is essential for infectious growth in the plant (Takano et al. 2000). When mycelial blocks of the cmk1 $\Delta$ strain DCM1 were inoculated, lesions were not formed (Fig. 6C). These results indicate that mycelial blocks can be used for infectious growth assay. When mycelial blocks of the $c p k 1 \Delta$ and $c a c 1:: h p h$ strains were inoculated onto wound sites on cucumber leaves, they formed no anthracnose lesions (Fig. 6A and B), but caused tiny yellow spots that were identical to those produced by wounding. Also, when mycelial blocks of the cpkl $\Delta$ and cacl::hph strains on minimal media were inoculated, identical results were obtained (data not shown). These results indicate that $C P K 1$ and $C A C 1$ are required for infectious growth in plants by $C$. lagenarium. The capacity for infectious growth of the SGC mutants also was investigated. Like the $c p k 1 \Delta$ strain, SGC1 (rpkld, cpk1-1) and SGC3 (rpk1 $\Delta, c p k 1-3$ ) did not form lesions (Fig. 6D and F). In contrast, SGC2 (rpk1 $1, c p k 1-2)$ frequently formed lesions through wound inoculation, although the lesions formed by SGC2 were smaller than those formed by the $p k s 1$ strain ALB1 (Fig. 6E).

\section{DISCUSSION}

cAMP signaling-mediated regulation of germination.

We have reported roles for the cAMP-PKA signaling pathway in the anthracnose fungus $C$. lagenarium. Functional analysis of $C P K 1$ and $C A C 1$ revealed that cAMP signaling in C. lagenarium is required for germination, appressorial penetration, and infectious growth. In other fungi, cAMP-signaling mutants have exhibited delayed germination. In $M$. grisea, the macl mutants, which lack adenylate cyclase, exhibit delayed germination, whereas the cpkA mutants, which lack the PKA catalytic sub- unit, germinate without delay (Choi and Dean 1997; Xu et al. 1997). The $C$. trifolii mutant lacking the catalytic subunit also exhibits a slight delay in germination (Yang and Dickman 1999). In contrast to delayed germination in the cAMP-signaling

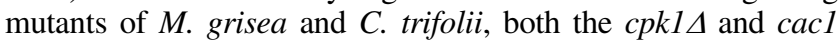
strains of $C$. lagenarium hardly germinated at all, even after long incubation. These results suggest that cAMP signaling is broadly involved in the germination of filamentous fungal pathogens and that this signaling plays essential roles in the germination of $C$. lagenarium. Incubation at low conidial density partially rescued the germination defects in the $c p k l$ and $c a c 1 \Delta$ strains. Inhibition of germination at high conidial densities is observed as "selfinhibition of germination" in many fungi. In several fungi such as $C$. gloeosporioides and $M$. grisea, it has been shown that chemical compounds called self-inhibitors are involved in this inhibition (Liu and Kolattukudy 1999; Tsurushima et al. 1995). Self-inhibition of conidial germination was observed in the wild-type strain (104-T) of $C$. lagenarium (i.e., the wild type germinates efficiently at $10^{5}$ conidia/ $\mathrm{ml}$ but fails to germinate at $10^{7}$ conidia/ml) (data not shown). The $c p k l \Delta$ and $c a c l$ strains failed to germinate at $10^{5} \mathrm{conidia} / \mathrm{ml}$ but germination frequency increased at $10^{4}$ conidia $/ \mathrm{ml}$, suggesting that these strains are more sensitive to self-inhibition effects. We consider that the failure of germination in these mutants is due partly to a self-inhibition mechanism, and that the cAMP-PKA pathway acts by overcoming this inhibitory mechanism. Conidial density had only slight effects on the germination defects of the $c m k 1 \Delta$ strains. It is possible that the putative self-inhibition mechanism inhibits the Cmk1-mediated germination pathway (Fig. 7). Fatty acids partly restored the germination of the $c p k 1 \Delta, c a c 1$, and $c m k 1 \Delta$ strains, implying that fatty acids induce germination in $C$. lagenarium. During infection-related morphogenesis, partial breakdown of lipid bodies is observed in conidia. The degradation of lipids to generate fatty acids and glycerol in the conidium might induce its germination (Fig. 7).

\section{Different phenotypes of cAMP-signaling mutants.}

The phenotype of the cpkl $\Delta$ strains was not identical to that of the $c a c l$ strains in growth, conidiation, or germination. This
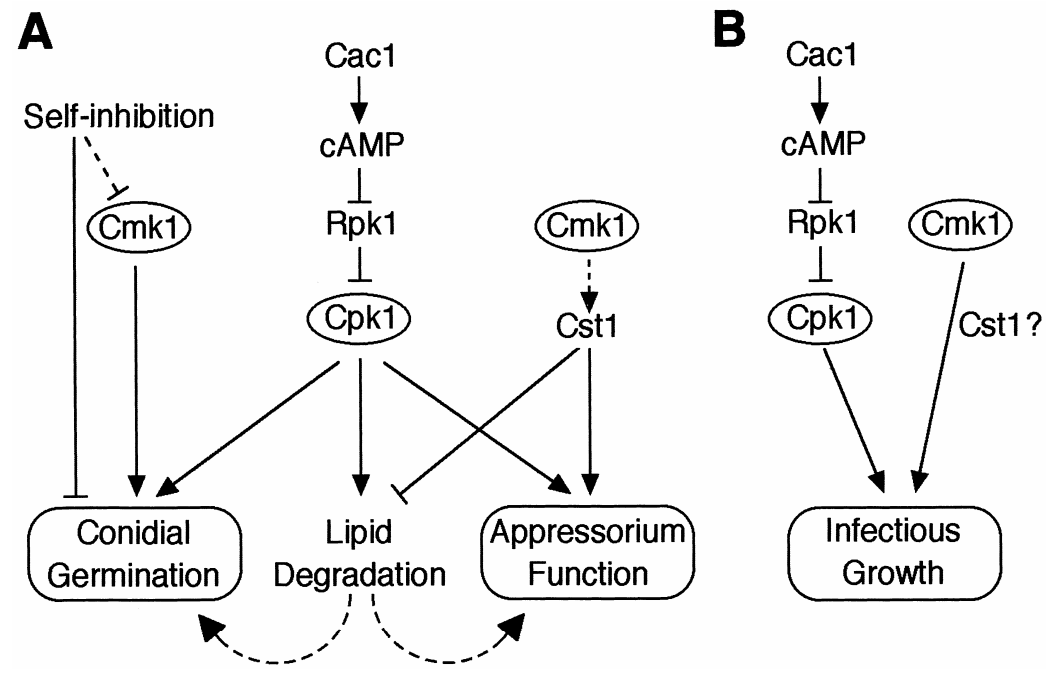

Fig. 7. Proposed model of the cyclic AMP (cAMP)-PKA and Cmk1 mitogen-activated protein kinase (MAPK) pathways in fungal infection of Colletotrichum lagenarium. A, cAMP-Cpk1 signaling is involved in germination and appressorial penetration. cAMP is produced by adenylate cyclase, Cac1, and activation of Cpk1 by cAMP induces degradation of lipids, whereas Rpk1 represses this degradation. Regulation of lipid degradation by PKA might play important roles in appressorium functionality. Lipid metabolism also might be involved in germination. Cmk1 MAPK is involved in germination and this pathway might be interrupted by a self-inhibition effect on germination at high conidial densities. Cst1, a putative down-stream target of Cmk1, is not essential for germination but is required for appressorial penetration. Cst1 negatively regulates lipid degradation (Tsuji et al. 2003). Dashed lines indicate hypothesized steps. B, cAMP signaling is essential for infectious growth. Cmk1 MAPK also is required for infectious growth. In contrast, the cst 1 mutant retained the capacity for infectious growth, although the cst1 mutant showed a slight reduction in infectious growth (Tsuji et al. 2003). 
suggests the presence of an unidentified cAMP-mediated regulatory pathway in addition to the Cpk1 pathway. S. cerevisiae has three different catalytic subunits of PKA (Toda et al. 1987). In M. grisea, the presence of an unidentified PKA in addition to CpkA has been suggested (Adachi and Hamer 1998). Thus, additional PKA-related kinases may exist in $C$. lagenarium. However, SGC1 (rpkla cpk1-1) lost most PKA activity, indicating that $C P K 1$ encodes the major PKA in $C$. lagenarium that could be assayed. Also, the phenotype of the cpkl $\Delta$ rpkl $\Delta$ strain is quite similar to that of the $c p k 1 \Delta$ strain, implying that $C$. lagenarium has no additional PKA catalytic subunits regulated by Rpk1. Therefore, we cannot exclude the possibility that cAMP regulates other aspects of cell metabolism distinct from the PKA-related pathways. Interestingly, growth rate, number of conidia, and germination ratio in three

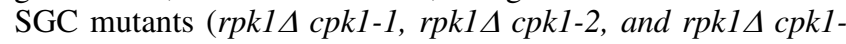
3) usually are higher than those of the $c p k 1 \Delta$ rpkl $\Delta$ strain, although the phenotypes of the SGC mutants are not identical. We consider that the mutated Cpk1 proteins in the SGC mutants make some contribution to the regulation of growth, conidiation, and germination, whereas this is impossible when Cpk1 is deleted. In both SGC1 and SGC3, mutations in Cpk1 occur in amino acid residues that are highly conserved in the serine/threonine protein kinases, whereas this is not the case in SGC2. Compared with SGC1 and SGC3, SGC2 germinates more efficiently and does not completely lose its capacity for infectious growth. However, SGC2 has a defect in appressorial penetration and is nonpathogenic on cucumber. This suggests the possibility that SGC2 retains PKA activity at a low level. For successful infection, $C$. lagenarium may require temporary high PKA activity, especially for appressorial penetration.

\section{Appressorium functionality and signaling pathways.}

Pharmacological analysis using cAMP and phenotypic analysis of the mac1 mutant indicated that cAMP signaling is required for appressorium formation in M. grisea (Choi and Dean 1997; Lee and Dean 1993). In contrast, germinating conidia of the cpkl and cacl strains formed melanized appressoria, indicating that cAMP signaling is not essential for the differentiation of germ tubes into appressoria in $C$. lagenarium. In contrast, the Mpk1-related MAPK is essential for appressorium formation in C. lagenarium but not in $M$. grisea (Kojima et al. 2002; Xu et al. 1998;). These data suggest that signaling cascades regulating appressorium formation could be diverse among fungal pathogens. We showed that cAMP signaling regulates lipid degradation in appressoria, and suggest a possibility that this regulation is involved in appressorium functionality. In $M$. grisea, $C P K A$-encoded PKA is involved in the regulation of lipid degradation and this metabolism produces glycerol that is thought to generate appressorial turgor (Thines et al. 2000). Pmk1 MAPK, which is related to yeast Fus3/Kss1 MAPKs, also has been shown to be involved in the transport of lipid reserves into appressoria, suggesting that MAPK is involved in lipid metabolism in $M$. grisea (Thines et al. 2000). In C. lagenarium, Cst1 is essential for appressorial penetration, and the appressoria of the cst $1 \Delta$ strain degraded lipids very rapidly, suggesting that Cst1 represses lipid degradation (Tsuji et al. 2003). Cst1 has high homology with yeast Ste12, which is a downstream target of Fus3/Kss1 MAPKs. Consistently, the cmkl $\Delta$ strains of $C$. lagenarium show rapid degradation of lipids (data not shown). Therefore, it is plausible that Cst1 functions downstream from Cmk1 (Fig. 7). However, the cst $1 \Delta$ strains retain the capacity for germination and infectious growth, unlike the cmkl $\Delta$ strains, implying that Cmk1 regulates an unidentified factor or factors required for these steps (Fig. 7).
cAMP signaling regulates fungal infection cooperatively with the Cmk1 MAPK cascade.

The Fus3/Kss1-related MAPKs, including Cmk1, are involved in the infectious-growth stage in fungal pathogens $(\mathrm{Xu}$ 2000). Phenotypic analysis of the $c p k l$ and cacl strains indicated that the cAMP-PKA signaling pathway is essential for infectious growth in planta by $C$. lagenarium. This result suggests that CAMP-PKA signaling cooperatively regulates infectious growth with Cmk1 MAPK in C. lagenarium (Fig. 7). Interestingly, agar-invasive or pseudohyphal growth of $S$. cerevisiae is controlled by both Kss1 MAPK and cAMP-PKA signaling (Pan et al. 2000). Involvement of Cmk1 and cAMP-Cpk1 signaling in infectious growth strongly suggests that there is a common regulatory mechanism between the agar-invasive growth of yeast and invasive growth in planta by fungal pathogens. In $S$. cerevisiae, the cAMP-PKA and MAPK pathways control the expression of Flo11, which is essential for invasive growth. In $U$. maydis, crosstalk between MAPK and cAMP signaling controls dimorphism and virulence (Mayorga and Gold 1999). Additionally, mating is regulated by the differential phosphorylation of the transcription factor Prf1 through MAPK and cAMP signaling (Kaffarnik et al. 2003). Cmk1 and Cpk1 might regulate a common factor essential for infectious growth in plants. Our purpose in this study was to understand the roles of the cAMP-PKA signaling pathway in fungal pathogenicity in detail. To extend our understanding of the cAMP-PKA signaling pathway, it will be necessary to identify the upstream and downstream factors in this pathway. The results of this study will contribute to our understanding of the relationship between signaling pathways and fungal pathogenesis, and to the design of strategies to protect plants from fungal infection.

\section{MATERIALS AND METHODS}

\section{Fungal strains and culture conditions.}

C. lagenarium strain 104-T (stock culture of the Laboratory of Plant Pathology, Kyoto University) was used as the wildtype strain. All $C$. lagenarium cultures were maintained on $3.9 \%$ (wt/vol) PDA (Difco Laboratories, Detroit) at $24^{\circ} \mathrm{C}$. Conidia were collected by gently scraping cultures incubated for 7 days. $\mathrm{MM}$ consisted of $\mathrm{NaNO}_{3}$ at $6 \mathrm{~g} /$ liter, $\mathrm{KCl}$ at 0.52 $\mathrm{g} /$ liter, $\mathrm{MgSO}_{4} \cdot 7 \mathrm{H}_{2} \mathrm{O}$ at $0.152 \mathrm{~g} /$ liter, $\mathrm{KH}_{2} \mathrm{PO}_{4}$ at $1.52 \mathrm{~g}$ liter, $0.001 \%$ thiamine, and $0.1 \%$ trace elements supplemented with glucose at $10 \mathrm{~g} /$ liter.

\section{Cloning of $C P K 1$ and $C A C 1$.}

$C P K 1$ and $C A C l$ of $C$. lagenarium were isolated by PCR using degenerate primers. For isolation of $C P K 1$, four degenerate primers, CPKFYA (5'-TTCTATGCNGTNAARGT3'), CPKNLY (5'-CCCAGAATTCAACCTGTAYAGGTNATG$3^{\prime}$ ), CPKWWS (5'-CAGGTTGCGGCCGCATTCCCAGNGA CCACCA- $3^{\prime}$ ) and CPKIYE (5'-CACAGCATYTCRTADAT$3^{\prime}$ ), were designed based on the conserved amino acid and nucleotide sequences of the PKA catalytic subunit in other organisms, including C. trifolii, M. grisea, S. cerevisiae, and U. maydis. CPKNLY contains a terminal EcoRI site and CPKWWS contains a terminal NotI site. The first PCR was performed with primers CPKFYA and CPKIYE. Nested PCR was performed with primers CPKNLY and CPKWWS. For the isolation of $C A C 1$, three degenerate primers, ACAFM $\left(5^{\prime}\right.$ CGGGATCCGARGGNGAYGCNTTYATG-3'), ACHWG (5'CGGAATTCCCCARTGNRYNCCCAT-3'), and ACGQI (5'ATYTGNCCNCCRTCNGC-3'), were designed from conserved sequences of the adenylate cyclase genes of other organisms. ACAFM contains a terminal BamHI site, whereas ACHWG contains a terminal EcoRI. The first PCR was performed with 
ACAFM and ACGQI, and nested PCR was performed with ACAFM and ACHWG. The cDNA synthesized from mRNA of conidia at the prepenetration stage was used as a template for PCR screening for $C P K 1$ and $C A C 1$. PCR conditions were as follows: 30 cycles of $94^{\circ} \mathrm{C}$ for $1 \mathrm{~min}, 55^{\circ} \mathrm{C}$ for $1 \mathrm{~min}$, and $72^{\circ} \mathrm{C}$ for $1 \mathrm{~min}$, with a final extension at $72^{\circ} \mathrm{C}$ for $5 \mathrm{~min}$. PCR products for CPKl were digested with EcoRI and NotI, whereas those for $C A C l$ were digested with $E c o$ RI and BamHI. Digested products were cloned into pBlueScript II $\mathrm{KS}-$ (Stratagene, La Jolla, CA, U.S.A.) and sequenced. Sequencing of the clones identified pCPK1 and pCAC1 exhibiting significant homology with the PKA catalytic subunit and adenylate cyclase, respectively. The insert fragments of pCPK1 and pCAC1 were used as probes to screen genomic clones containing $C P K 1$ and $C A C 1$, respectively, from a $C$. lagenarium cosmid library. The DNA sequences of the $C P K 1$ and $C A C 1$ genes were determined using a Big Dye Terminator Cycle Sequencing Ready Reaction kit (Applied Biosystems, Warrington, U.K.) and an automated DNA sequencer (Applied Biosystems Model 310). To compare the sequences of CPK1 in the wildtype and SGC mutants, a 2.2-kb genomic fragment containing $C P K 1$ was amplified from the genomes of tested strains using primers CPK1S (5'-CTTGAATCGACGAATCACTTTG-3') and CPK1AS (5'-GCAAGTGCAGACATGACCGAG-3'). Amplified PCR products were subjected to direct sequencing. As for RTPCR analysis of $C P K 1$ in the wild type and SGC1, cDNA was synthesized using mRNA derived from mycelia of each strain grown in PSY (liquid extract from $200 \mathrm{~g}$ of potato, $20 \mathrm{~g}$ of sucrose, and $2 \mathrm{~g}$ of yeast extract per liter) and was used as a template. The first PCR was performed with CPK1RTS1 (5' ATGCCTACCCTCGGCTTCCTCAAG-3') and CPK1RTAS1 (5'-TTAGAAGTCGGTGAATAGGTGGCC-3'), and nested PCR was performed with CPK1RTS2 (5'-TAGATGGTGATGG ACTTCGTCGAG-3') and CPK1RTAS2 (5'-GAAGTCGGTGA ATAGGTGGCCATA-3').

\section{Plasmid construction.}

For a complementation assay of SGC1 with $C P K 1$, an approximately $3.0-\mathrm{kb} K p n \mathrm{I}-\mathrm{Sal \textrm {I }}$ genomic fragment containing $C P K 1$ was isolated from a $C P K 1$ cosmid clone, and introduced into the KpnI-SalI sites of pCB1531, which carries the bar gene (Sweigard et al. 1997), generating pCBCPK1. To construct the gene-replacement vector pGDCPK1, a 5.0-kb fragment containing the $3^{\prime}$ flanking region of $C P K 1$ was amplified from pCBCPK1A carrying the 6.2-kb $A p a \mathrm{I}$ fragment at the CPK1 locus, using PCR with primers CPK1D1 (5'-TGCGC TCGTCCATCTTCTTGC-3') and CPK1D2 (5'-GAAGGGCC CTCTCGCCTAGACAGAAGATG-3'). The primer CPK1D2 contains a terminal ApaI site. The amplified fragment was digested with $A p a \mathrm{I}$ and $K p n \mathrm{I}$, and the resulting 2.0-kb ApaI$K p n I$ fragment containing the $3^{\prime}$ flanking region of $C P K 1$ was introduced into the ApaI-KpnI sites of pCB1636 to produce plasmid pCB3CPK1. The 2.5-kb fragment containing the 5' flanking region of $C P K 1$ was amplified from pCBCPK1S, which carries the 5.2-kb SalI fragment containing $C P K 1$, using PCR with CPK1D3 (5'-GGACTAGTTTGCCCGTTGAGGAG AGT-3') and the M13 forward primer. The primer CPK1D3 contains a terminal SpeI site. The amplified fragment was digested with SpeI, and the SpeI-digested fragment was introduced into the SpeI site of pBluescript to generate the plasmid pBS5CPK1. pBS5CPK1 was digested with EcoRI, and the resulting $1.0-\mathrm{kb}$ fragment containing the $5^{\prime}$ flanking region of CPK1 was introduced into the EcoRI site of pCB3CPK1 to generate the plasmid pGDCPK1. CAC1 was mutated using an adaptation of the TAG-KO method. The TrpC/hph gene in pCB1636 was introduced into the EcoRV site of GPS3, which generated pGPSHPH. pGPSHPH contains the hygromycin-resistance gene between Tn7-based transposon ends (GPS-HYG). The GPS-HYG element was mobilized using the TnsABC transposase complex in vitro. A cosmid clone pD4CAC1 containing $C A C l$ was used as the target. The sequences of the transposon-inserted regions were determined using primer TN7L (5'-ATAATCCTTAAAAACTCCATTTCC ACCCCT-3'). An insertion was formed within the coding region of $C A C 1$ in the plasmid pKOCAC1. We also produced a TAG$\mathrm{KO}$ construct for $P K S 1$, which is involved in melanin biosynthesis, using a cosmid clone containing PKS1. In the plasmid pKOPKS1, GPS-HYG was inserted into the coding region of PKS1 (at 1,578 amino acids). To evaluate the TAG-KO system in C. lagenarium, we first introduced pKOPKS1 into the C. lagenarium wild type. As a result, 32 of 34 hygromycin-resistant transformants produced colonies, identical to the $p k s 1$ mutant 79215 previously identified. This experiment demonstrated high frequency of gene disruption in $C$. lagenarium using TAG-KO.

\section{Fungal transformation.}

Preparation of protoplasts and transformation of $C$. lagenarium were performed according to a method described previously (Takano et al. 2001a). Hygromycin- and bialaphosresistant transformants were selected on regeneration medium containing hygromycin B $(100 \mu \mathrm{g} / \mathrm{ml})$ (Wako Pure Chemicals, Osaka, Japan) and bialaphos $(250 \mu \mathrm{g} / \mathrm{ml}$ ) (provided by $\mathrm{N}$. Fuchigami, Meiji Seika), respectively.

\section{Genomic DNA blot analysis.}

Total DNA of $C$. lagenarium was isolated from mycelia using the DNeasy Plant Mini Kit (Qiagen, Hilden, Germany) according to the manufacturer's instructions. DNA digestion, gel electrophoresis, labeling of probes, and hybridization were performed according to the manufacturer's instructions and standard methods (Sambrook et al. 1989). DNA probes were labeled with digoxygenin-dUTP (Roche Diagnostics Japan, Tokyo) with the BcaBEST digoxygenin labeling kit (Takara, Ohtsu, Japan). Hybridization was performed as described previously (Takano et al. 1997).

\section{Pathogenicity test.}

Conidia were collected from 7-day-old PDA cultures and suspended in water (approximately $5 \times 10^{5}$ conidia $/ \mathrm{ml}$ ). Conidial suspensions were spotted onto the surfaces of detached cucumber leaves (Cucumis sativus L. 'Suyo'). Inoculated leaves were placed in humid petri dishes and incubated at $25^{\circ} \mathrm{C}$ for 7 days. For microscopy, $20 \mu \mathrm{l}$ of conidial suspension was spotted onto the lower epidermis of cucumber cotyledons. The epidermal layers were peeled off and observed under light microscopy $24 \mathrm{~h}$ after inoculation. To investigate penetration, inoculated cotyledons were incubated for 4 days and stained with lactophenol aniline blue (Takano et al. 1997). For leaf wound inoculations, wounds ( 1 to $2 \mathrm{~mm}^{2}$ ) were produced by scratching leaves with $26 \mathrm{G}_{1 / 2}$ needles, and leaves were inoculated with mycelial blocks at the wound sites.

\section{Microscopy.}

Conidia of Colletotrichum lagenarium were harvested from 7-day-old cultures on PDA. Conidial attachment was assayed by the method described previously (Takano et al. 2000). As for germination and appressorium-formation assays, conidia were suspended in water, $0.1 \%$ yeast extract, $50 \mathrm{mM}$ cAMP, or $0.5 \%$ Tween 80 solutions. Each conidial suspension $\left(10^{5}\right.$ conidia or $10^{4}$ conidia/ml) was placed in a $3.8-\mathrm{cm}$-diameter glass petri dish and incubated at $24^{\circ} \mathrm{C}$. Images were captured with a chilled charge-coupled device camera (Argus50; Hamamatsu Photonics, Hamamatsu, Japan). To stain lipids with Nile Red, 
conidia were incubated on glass slides (Takano et al. 2001b) and stained with Nile Red solution (Greenspan et al. 1985; Weber et al. 1999) at $2.5 \mu \mathrm{g} / \mathrm{ml}$. For incorporation of Nile Red into appressoria, conidia were incubated with carpropamid at $10 \mu \mathrm{g} / \mathrm{ml}$ (Hattori et al. 1994). Images were captured with a laser scanning microscope (Fluoview FV500; Olympus, Tokyo). Relative fluorescence intensity of Nile-Red-strained lipids in appressoria was measured at intervals of $2 \mu \mathrm{m}$ for five slices using a rhodamine-phalloidin filter, and total fluorescence intensity was calculated by adding together the fluorescence values of each slice.

\section{ACKNOWLEDGMENTS}

This work was supported in part by a Grant-in-Aid (15028210) for Scientific Research on Priority Area (A) from the Ministry of Education, Culture, Sports, Science and Technology, Japan and a Grant-in-Aid (15780035) for Young Scientists (B), and a Grant-in-Aid (13306005) for Scientific Research (A) from the Japan Society of the Promotion of Science. We thank K. Kojima and K. Mise for valuable suggestions and support during the course of this work.

\section{LITERATURE CITED}

Adachi, K., and Hamer, J. E. 1998. Divergent cAMP signaling pathways regulate growth and pathogenesis in the rice blast fungus Magnaporthe grisea. Plant Cell 10:1361-1373.

Agrios, G. N. 1988. Plant Pathology. 3rd ed. Academic Press, San Diego, CA, U.S.A.

Choi, W., and Dean, R. A. 1997. The adenylate cyclase gene MAC1 of Magnaporthe grisea controls appressorium formation and other aspects of growth and development. Plant Cell 9:1973-1983.

Gold, S. E., Brogdon, S. M., Mayorga, M. E., and Kronstad, J. W. 1997. The Ustilago maydis regulatory subunit of a cAMP-dependent protein kinase is required for gall formation in maize. Plant Cell 9:15851594.

Gold, S. E., Duncan, G., Barrett, K., and Kronstad, J. W. 1994. cAMP regulates morphogenesis in the fungal pathogen Ustilago maydis. Genes Dev. 8:2805-2816.

Greenspan, P., Mayer, E. P., and Fowler, S. D. 1985. Nile red: A selective fluorescent stain for intracellular lipid droplets. J. Cell. Biol. 100:965-973.

Hamer, L., Adachi, K., Montenegro-Chamorro, M. V., Tanzer, M. M., Mahanty, S. K., Lo, C., Tarpey, R. W., Skalchunes, A. R., Heiniger, R. W., Frank, S. A., Darveaux, B. A., Lampe, D. J., Slater, T. M., Ramamurthy, L., Dezwaan, T. M., Nelson, G. H., Shuster, J. R., Woessner, J., and Hamer, J. E. 2001. Gene discovery and gene function assignment in filamentous fungi. Proc. Natl. Acad. Sci. U.S.A. 98:5110-5115.

Hanks, S. K., and Quinn, A. M.1991. Protein kinase catalytic domain sequence data base: Identification of conserved features of primary structure and classification of family members. Methods Enzymol. 200:38-62.

Hattori, T., Kurahashi, K., Kagabu, S., Konze, J., and Kraatz, U. 1994. KTU3616: A novel fungicide for rice blast control. Pages 517-524 in: Abstr. Brighton Crop Prot. Conf. Pest Dis. The British Crop Protection Council, Hampshire, U.K.

Ishida, N., and Akai, S. 1969. Relation of temperature to germination of conidia and appressorium formation in Colletotrichum lagenarium. Mycologia 61:382-386.

Kaffarnik, F., Muller, P., Leibundgut, M., Kahmann, R., and Feldbrugge, M. 2003. PKA and MAPK phosphorylation of Prf1 allows promoter discrimination in Ustilago maydis. EMBO (Eur. Mol. Biol. Organ.) J. 22:5817-5826.

Kojima, K., Kikuchi, T., Takano, Y., Oshiro, E., and Okuno, T. 2002. The mitogen-activated protein kinase gene $M A F 1$ is essential for the early differentiation phase of appressorium formation in Colletotrichum lagenarium. Mol. Plant-Microbe Interact. 15:1268-1276.

Kronstad, J. W. 1997. Virulence and cAMP in smuts, blasts and blights. Trends Plant Sci. 2:193-199.

Lee, N., D'Souza, C. A., and Kronstad, J. W. 2003. Of smuts, blasts, mildews, and brights: cAMP signaling in phytopathogenic fungi. Annu. Rev. Phytopathol. 41:399-427.

Lee, Y.-H., and Dean, R. A. 1993. cAMP regulates infection structure formation in the plant pathogenic fungus Magnaporthe grisea. Plant Cell 5:693-700.
Lee, Y.-H., and Dean, R. A. 1994. Hydrophobicity of contact surface induces appressorium formation in Magnaporthe grisea. FEMS (Fed. Eur. Microbiol. Soc.) Microbiol. Lett. 115:71-76.

Liu, Z. E., and Kolattukudy, P. E. 1999. Early expression of the calmodulin gene, which precedes appressorium formation in Magnaporthe grisea, is inhibited by self-inhibitors and requires surface attachment. J. Bacteriol. 181:3571-3577.

Mayorga, M. E., and Gold, S. E. 1999. A MAP kinase encoded by the $u b c 3$ gene of Ustilago maydis is required for filamentous growth and full virulence. Mol. Microbiol. 34:485-97.

Mitchell, T. K., and Dean, R. A. 1995. The cAMP-dependent protein kinase catalytic subunit is required for appressorium formation and pathogenesis by the rice blast pathogen Magnaporthe grisea. Plant Cell 7:1869-1878

Pan, X., Harashima, T., and Heitman, J. 2000. Signal transduction cascades regulating pseudohyphal differentiation of Saccharomyces cerevisiae. Curr. Opin. Microbiol. 3:567-572.

Sambrook, J., Fritsch, E. F., and Maniatis, T. 1989. Molecular Cloning: A Laboratory Manual. 2nd ed. Cold Spring Harbor Laboratory Press, Cold Spring Harbor, NY, U.S.A.

Sweigard, J., Chumley, F., Carroll, A., Farrall, L., and Valent, B. 1997. A series of vectors for fungal transformation. Fungal Genet. Newsl. 44:52-55.

Takano, Y., Kubo, Y., Shimizu, K., Mise, K., Okuno, T., and Furusawa, I. 1995. Structural analysis of $P K S 1$, a polyketide synthase gene involved in melanin biosynthesis in Colletotrichum lagenarium. Mol. Gen. Genet. 249:162-167.

Takano, Y., Kubo, Y., Kawamura, C., Tsuge, T., and Furusawa, I. 1997. The Alternaria alternata melanin biosynthesis gene restores appressorial melanization and penetration of cellulose membranes in the melanindeficient albino mutant of Colletotrichum lagenarium. Fungal Genet. Biol. 21:131-140.

Takano, Y., Kikuchi, T., Kubo, Y., Hamer, J. E., Mise, K., and Furusawa, I. 2000. The Colletotrichum lagenarium MAP kinase gene CMKI regulates diverse aspects of fungal pathogenesis. Mol. Plant-Microbe Interact. 13:374-383.

Takano, Y., Komeda, K., Kojima, K., and Okuno, T. 2001a. Proper regulation of cyclic AMP-dependent protein kinase is required for growth, conidiation, and appressorium function in the anthracnose fungus Colletotrichum lagenarium. Mol. Plant-Microbe Interact. 14:1149-1157.

Takano, Y., Oshiro, E., and Okuno, T. 2001b. Microtubule dynamics during infection-related morphogenesis of Colletotrichum lagenarium. Fungal Genet. Biol. 34: 107-121.

Thines, E., Weber, R. W., and Talbot, N. J. 2000. MAP kinase and protein kinase A-dependent mobilization of triacylglycerol and glycogen during appressorium turgor generation by Magnaporthe grisea. Plant Cell 12:1703-1718.

Thompson, J. D., Higgins, D. G., and Gibson, T. J. 1994. CLUSTALW: Improving the sensitivity of progressive multiple sequence alignment through sequence weighting, position-specific gap penalties and weight matrix choice. Nucleic Acids Res. 22:4673-4680.

Toda, T., Cameron, S., Sass, P., Zoller, M., and Wigler, M. 1987. Three different genes in $S$. cerevisiae encode the catalytic subunits of the cAMP-dependent protein kinase. Cell 50:277-287.

Tsuji G., Fujii, S., Tsuge, S., Shiraishi, T., and Kubo, Y. 2003. The Colletotrichum lagenarium Ste12-like gene CST1 is essential for appressorial penetration. Mol. Plant-Microbe Interact. 16:315-325.

Tsurushima, T., Ueno, T., Fukami, H., Irie, H., and Inoue, M. 1995. Germination self-inhibitors from Colletotrichum gloeosporioides f. sp. jussiaea. Mol. Plant-Microbe Interact. 8:652-657.

Weber, R. W. S., Wakley, G. E., and Pitt, D. 1999. Histochemical and ultrastructural characterization of vacuoles and spherosomes as components of the lytic system in hyphae of the fungus Botrytis cinerea. Histochem. J. 31:293-301.

Xu, J.-R. 2000. MAP kinases in fungal pathogens. Fungal Genet. Biol. 31:137-152.

Xu, J.-R., Urban, M., Sweigard, J. A., and Hamer, J. E. 1997. The CPKA gene of Magnaporthe grisea is essential for appressorial penetration. Mol. Plant-Microbe Interact. 10:187-194.

Xu, J.-R., Staiger, C. J., and Hamer, J. E. 1998. Inactivation of the mitogen-activated protein kinase Mps1 from the rice blast fungus prevents penetration of host cells but allows activation of plant defense responses. Proc. Natl. Acad. Sci. U.S.A. 95:12713-12718.

Yang, Z., and Dickman, M. B. 1999. Colletotrichum trifolii mutants disrupted in the catalytic subunit of cAMP-dependent protein kinase are nonpathogenic. Mol. Plant-Microbe Interact. 12:430-439. 